Methods Arthritis was induced by collagen-induced arthritis (CIA) and passive collagen antibody induced arthritis (CAIA) in respectively $\mathrm{C} 57 \mathrm{BL} / 6$ and RAG2-/- (T- and B-cell deficient) mice. Animals were subjected to different regimens of mechanical strain. Increased strain occurred in voluntary running mice whereas tail suspension (unloading) abolished mechanical strain; both were compared to control housing conditions. The impact of different loading conditions was measured on clinical disease score, histology, micro-CT images and erosion quantification, gene induction in tendon and synovial tissue, immune cell recruitment in situ, development of anti-collagen antibodies and their pattern of siaylation and galactosylation.

Results Voluntary running of CIA in C57BL/6 mice markedly induced an early onset and increased progression whereas no disease onset could be observed in the hind paws from animals in unloaded conditions. CAIA in running RAG2-/- mice also induced early arthritic symptoms and severe progression. Intriguingly, running conditions were sufficient to induce arthritis without the need of LPS as an inflammatory trigger. Mechanical strain did not alter however IgG autoantibody levels nor their levels of galactosylation and sialylation. Furthermore, we demonstrate that mechanical strain on stromal cells results in recruitment of classical monocytes into specialised mechano-sensitive regions characterised by a unique microanatomy. This promotes local inflammation and differentiation into local osteoclasts which induce regional erosions. A striking similarity was observed in the pattern of joint erosions in human patients with RA and SpA which were also confined to these mechanosensitive regions.

Conclusions This study provides the first evidence that mechanical strain controls the transition from systemic autoimmunity into site-specific joint inflammation. Homing of inflammation and development of erosions was confined to mechano-sensitive regions, characterised by a high number of attachment- and contact points for tendons. This represents a novel paradigm and explains why arthritis in mice and humans is characterised by a regional and patchy distribution. Curiously, this pathway does not rely on adaptive immunity but rather on stromal cells. Mechanostimulation of mesenchymal cells induced CXCL1 and CCL2 permitting recruitment of classical monocytes which can differentiate into bone-resorbing osteoclasts. Thus, mechanical strain controls the site-specific direction of inflammation and tissue damage in arthritis.

Disclosure of interest None declared

\section{P001 MECHANISMS OF BONE EROSION AND PAIN TRIGGERED BY ANTIBODIES TARGETING POST- TRANSLATIONAL PROTEIN MODIFICATIONS IN RHEUMATOID ARTHRITIS}

\begin{abstract}
${ }^{1} \mathrm{~A}$ Krishnamurthy ${ }^{*},{ }^{2} \mathrm{~K}$ Sandor, ${ }^{2} \mathrm{~A}$ Jurczak, ${ }^{3} \mathrm{MJ}$ Andrade, ${ }^{1} \mathrm{~J}$ Steen, ${ }^{1} \mathrm{C}$ Grönwall, ${ }^{1} \mathrm{G}$ Wigerblad, ${ }^{1} \mathrm{P}$ Titcombe, ${ }^{1} \mathrm{H}$ Wähämaa, 'B Rethi, ${ }^{2} \mathrm{C}$ Svennson, ${ }^{1} \mathrm{~A}$ Catrina. ${ }^{1}$ Medicine, Rheumatology Unit; ${ }^{2}$ Department of Physiology and Pharmacology, Karolinska Institutet, Stockholm, Sweden; ${ }^{3}$ Department of Unidad Academica Multidisciplinaria Reynosa Aztlan, UniversidadAutonoma de Tamaulipas, Reynosa, Tamaulipas, Mexico
\end{abstract}

\subsection{6/annrheumdis-2018-EWRR2018.28}

Introduction Pain and bone loss are cardinal features of rheumatoid arthritis (RA), which can be triggered by anti-citrullinated protein antibodies (ACPAs).

Objectives We aimed to investigate whether ACPA fine specificities could influence osteoclast (OC) induction and we studied the contribution of ACPA $\mathrm{Fc}$ and $\mathrm{Fab}$ regions in the regulation of OCs. We also investigated whether in vivo targeting of OCs by bis-phosponate could influenceACPA-induced pain in mice.

Methods Polyclonal ACPAs and control IgGs were purified from the peripheral blood of RA patients using protein $\mathrm{G}$ and anti-cyclic citrullinated peptide (CCP)-2 affinity chromatography. Monoclonal ACPA and rheumatoid factor (RF) IgGs were generated from single synovial plasma cells or antigen tetramer-sorted peripheral blood memory B-cells. OCs were generated from CD14 + monocytes of healthy individuals or bone marrow cells of FcgRIII or FcRg chain knockout mice in the presence of the various antibodies. OC differentiation was monitored by counting TRAP-positive multinucleated cells or in bone erosion assays. Mechanical hypersensitivity was assessed over time by Von Frey filaments and the up-down method in female adult wild type or FcRg chain knockout mice injected i.v. with polyclonal or monoclonal ACPA or control antibodies. Bone density was measured by micro-CT. Zoledronate $(100 \mathrm{ug} / \mathrm{kg})$ was injected i.p every third day to examine if blocking osteoclast activation alter ACPAinduced pain.

Results Polyclonal and two out of the nine tested monoclonal ACPAs increased osteoclastogenesis. Addition of a monoclonal RF antibody to OC cultures could not influence osteoclastogenesis in itself, but it significantly increased the effect of ACPAs. Dimeric Fab fragments prepared from polyclonal ACPAs could increase OC numbers similarly as the intact antibodies, suggesting a crucial role of the cell surface antigens triggered by ACPA binding in mediating the effects of these antibodies. On the other hand, whereas ACPAs increased osteoclastogenesis from bone marrow precursors of wild type mice, no stimulatory effects could be observed when bone marrow cells of FcgRIII or FcRg chain knockout mice were used, suggesting that $\mathrm{Fc}$ receptors might also be important for ACPA-mediated OC stimulation. Polyclonal and monoclonal ACPA induced pronounced mechanical hypersensitivity lasing for at least 3 weeks and injection of polyclonal and certain combinations of monoclonal ACPA lead to bone erosion detectable by micro-CT- Bisphosphonate treatment and FcRg chain depletion prevented development of pain-like behaviour in those groups.

Conclusions We demonstrated that ACPAs with certain specificities have the capacity to increase osteoclastogenesis whereas most of the tested clones showed no effect on OCs. The mechanism triggered by ACPA binding to developing OCs was mediated through both Fc-dependent and independent signals. ACPA-mediated pain hypersensitivity was dependent both on osteoclast activity and Fcg receptors.

Disclosure of interest None declared

\section{P002 HOW DO GLYCANS AFFECT IMMUNE CELLS IN RHEUMATOID ARTHRITIS?}

${ }^{1} \mathrm{~A}$ Molhoek*, ${ }^{2} \mathrm{~L}$ Hafkenscheid, ${ }^{2} \mathrm{R}$ Toes, ${ }^{1} \mathrm{~S}$ van Vliet, ${ }^{1} Y$ van Kooyk. ${ }^{1} M C B I$, VUmc, Amsterdam; 'UMC, Leiden, Netherlands

10.1136/annrheumdis-2018-EWRR2018.29

Introduction Anti-Citrullinated Protein Antibodies (ACPAs) are specific for Rheumatoid Arthritis (RA) and have been implicated in disease pathogenesis. The fragment antigen-binding domain (Fab) of ACPA was recently shown to be extensively glycosylated. ${ }^{1}$ It is known that glycans play a key role in controlling innate and adaptive immunity, ${ }^{2}$ however to date there 